\title{
Research Paper: Validity and Reliability of OARS Multidimensional Functional Assessment Questionnaire in Iranian Elderly
}

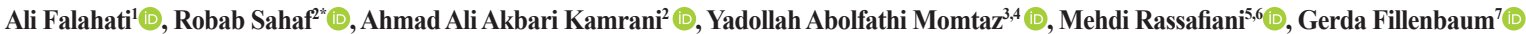

1. Department of Aging, University of Social Welfare and Rehabilitation Sciences, Tehran, Iran

2. Iranian Research Center on Aging, Department of Aging, University of Social Welfare and Rehabilitation Sciences, Tehran, Iran.

3. Department of Gerontology, University of Social Welfare and Rehabilitation Sciences, Tehran, Iran.

4. Malaysian Research Institute on Ageing (My Ageing), Universiti Putra Malaysia, Serdang, Selangor, Malaysia.

5. Department of Occupational Therapy, Faculty of Allied Health Sciences, Kuwait University, Kuwait City, Kuwait.

6. Pediatric Neurorehabilitation Research Center, University of Social Welfare and Rehabilitation Sciences, Tehran, Iran

7. Center for the Study of Aging and Human Development, Durham, North Carolina, USA.

Article info:

Received: 28 Nov 2017

Accepted: 10 Feb 2018

Keywords:

Psychometric, Geriatric assessment, Aging Iran, Translation
Cittation: Falahati A, Sahaf R, Akbari Kamrani AA, Abolfathi Momtaz Y, Rassafiani M, Fillenbaum G. Validity and Reliability of OARS Multidimensional Functional Assessment Questionnaire in Iranian Elderly. Iranian Rehabilitation Journal. 2018; 16(2):169-176. http://dx.doi.org/10.32598/irj.16.2.169

http://dx.doi.org/10.32598/irj.16.2.169

* Corresponding Author:

Robab Sahaf, PhD

Address: Iranian Research Center on Aging, Department of Aging, University of Social Welfare and Rehabilitation Sciences, Tehran, Iran.

Tel: +98 (912) 8204187

E-mail: robabsahaf@gmail.com 


\section{Introduction}

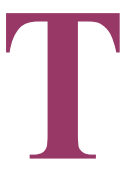

he worldwide elderly population is growing rapidly due to various factors such as improvement in sanitary and health conditions, improved medical care, reduced mortality rates, and increased birth rates.

Thus, this population has increased from 200 million in 1950 to 600 million in 2012, and the figure is estimated to reach 2 billion by 2050 [1]. According to 2016 census in Iran, $9.2 \%$ of the Iranian population (about 7 million) were $>60$-year-old [2]. With increasing age, people tend to experience reduction in all their functioning abilities [3]. The International Classification of Functioning, Disability, and Health (ICF) is a widely used framework for classifying and describing the information regarding health, disability, and functioning. ICF is a conceptual basis and utilized standard language for describing and measuring the health and disability. According to this classification system, function refers to the body functions, activities, and participation, while disability refers to the impairments and limitations in the activity and participation [4].

The quality of life and general health may be influenced negatively by reduced functioning [5]. Therefore, the health and quality of life of aging individuals as well as their level of functioning should be assessed regularly in order to plan appropriate interventions for their improvement [6]. Since different domains are involved in the functioning of older adults, instruments capable of concurrent assessment of all the different domains of functioning are essential. Some questionnaires that can be used to assess the functional ability include Katz index [7], Barthel index [8], Lawton [9], World Health Organization Disability Assessment Schedule (WHODAS) 2.0 [10], and care dependency scale [11] in the elderly. Although all these tools can assess the functioning in a specific domain based on the researcher's objective, none of them can concurrently assess the functioning in different domains (except WHODAS 2.0 that does not assess the economic and physical health domains), thereby indicating the advantage of MFAQ/OARS (multidimensional functional assessment questionnaire/older Americans resources and services).

It seems to be an appropriate assessment addressing five domains of functioning specifically designed for elderly, which includes social resources, economic resources, physical health resources, mental health resources, and selfcare capacity (activities of daily living) in part A, while part B evaluates the level of using the services [12]. Each domain of functioning receives a score ranging from 1 (highest level of functioning) to 6 (total impairment in the functioning). The total functioning score is calculated based on the performance in all domains, and ranges from 5 (highest level of functioning) to 30 (total impairment in the functioning) [12].

The MFAQ/OARS has been translated into at least to eight languages such as Dutch, Italian, Australian English, English, Swedish, Spanish, Portuguese, and Brazilian. The social resources domain has been validated by a joint study in The Netherlands, Italy, Austria, England, and Sweden [13], and the mental health domain has been validated in Brazil [14]; all the five domains have been validated in Spain [15] and Portugal [16]. Overall, the main version and the other forms of the questionnaire in various languages have shown satisfactory validity and reliability [13-16]. Given that old age is a critical stage of life and that the elderly population is growing continuously in Iran, the limitations of using non-validated tools due to linguistic, cultural, and geographical differences led the present study to examine the psychometric properties of the Persian version of MFAQ/OARS in Iranian elderly. To the best of our knowledge, the questionnaire is the only multidimensional tool that assesses the function among older individuals. This study would develop an appropriate tool for the comprehensive evaluation of the elderly and provide the basic information that would the healthcare policy makers and clinicians to plan interventions.

\section{Methods}

The present study is a non-experimental, psychometric study, and prior permission was obtained from the author of the MFAQ/OARS. Then, according to the IQOLA protocol [17], two individuals proficient in the English language translated the questionnaire into Persian. After making the necessary revisions, the other two translators back-translated the questionnaire into English. The items not compatible with the Iranian culture were replaced. Next, the questionnaire was sent to the author, who approved the consistency between the two forms of the questionnaire. Subsequently, the validity and reliability of the Persian version of the MFAQ/OARS were examined. In order to assess the face validity, the questionnaire was sent to 18 experts for their opinion on the quality of translation, comprehensibility, and appropriateness for the Iranian population. These highly experienced professionals belonged to different disciplines, including gerontology [7], occupational therapy [7], psychology [3], and physiotherapy [1], and their average age was $44.5 \pm 7.11$ years. 
Two indices were used to assess the content validity quantitatively: Content Validity Ratio (CVR) and Content Validity Index (CVI). According to the Lawshe table for determining the minimum CVR, items with CVRs $\geq 0.42$ (based on the opinions of 18 experts) were maintained [18]. The CVI based on the Bausell-Waltz method was set as $>79 \%$ [19]. In the next step, a total of 482 adults $>60$-year-old, attending the Comprehensive Healthcare Centers in Kashan, Iran, participated in this study. The inclusion criteria were as follows: age $>60$ years, the absence of cognitive disorders, and informed consent obtained for participation in the study. The study objectives were explained to the participants by the researchers, and the questionnaires completed by interviews. All statistical analyses were performed using SPSS version 23. In all the stages of completing the questionnaire, ethical considerations were addressed. Only those who were interested in participating in the study were recruited, the interviews did not hurt the participants or cost them anything, and the participants were allowed to leave the study at any time. This article was extracted from a Master's thesis (Number: 941193014) by the first author and approved by the Ethics Committee at the University of Social Welfare and Rehabilitation Sciences (Number: IR.USWR.REC.1395.235).

In order to assess the concurrent validity of MFAQ/ OARS, the WHODAS 2.0 validated in Iran by Abedzadeh et al. [20] and geriatric depression scale (GDS15) validated in Iran by Malakuti et al. [21] were also completed by the participants. The original form of the WHODAS 2.0 was developed by the WHO in 1988 based on ICF to assess the respondents' activities, limitations, and participation limitations [10]. It is a general tool for assessing the health and disability and can be used for both patients and the general population. The WHODAS 2.0 assesses the following domains: cognition (understanding and communicating), mobility, self-care, getting along (interacting with other people), life activities, and participation (joining in community activities); The complete form of this instrument comprises of 36 items [10]. The original form of GDS-15 was developed by Yesavage et al., and the long form of this questionnaire consisted of 30 items. In the present study, the 15-item version of GDS-15 was utilized [22]. Then, the correlations between the scores on the MFAQ/ OARS and WHODAS 2.0 and GDS-15 were estimated using the Spearman's rank correlation coefficient.

In addition, the inter-rater reliability was assessed. First, the raters were trained, and then, they independently provided their ratings. The correlation coefficient between the ratings and the Intra-class Correlation Coefficient
(ICC) was used to assess the inter-rater reliability. In order to assess the test-retest reliability, the MFAQ/OARS was completed by 20 elderly from the sample on two occasions at an interval of 2 weeks, and the consistency was assessed using the ICC.

\section{Results}

A total of 482 adults, aged $>60$ (ranged 60-96, mean $=71.74 \pm 9.82$ ) years, participated in the present study. The participants'). The cohort comprised of 269 (55.8\%) males and $213(44.2 \%)$ females. 95.6\% of the participants had an education level lower than that of high school diploma, 87.2\% were Persian, and 34\% lived alone. Table 1 summarized the demographic characteristics of the participants.

The average time of completing the questionnaire was $51.14 \pm 7.01 \mathrm{~min}$. Regarding face validity, all the $10 \mathrm{ex}-$ perts reported the quality of translation, comprehensibility, and appropriateness of the MFAQ/OARS for the Iranian population as acceptable. The examination of the content validity indicated that 2 items had a $C V R<0.42$ and 2 other items had a $\mathrm{CVI}<0.79$; these items were maintained in the questionnaire after making minor modifications for compatibility with the Iranian culture. The concurrent validity of the MFAQ/OARS was assessed by concurrent administration of WHODAS 2.0 and GDS-12 and calculating the Spearman's rank correlation coefficient for each domain of functioning. The results were presented in Table 2. The inter-rater reliability for each domain of functioning was also examined (Table 3). According to the results, there was a significant relationship observed, which indicated satisfactory inter-rater reliability.

The ICC indicated a significant agreement between the first and second stage of the questionnaire $(\mathrm{P}<0.0001)$, thereby demonstrating the replicability of the scores on the domains and subdomains of the MFAQ/OARS and its total score. The ICC for each domain of functioning was also demonstrated in Table 4.

In the present study, the psychometric properties of the Persian version of the MFAQ/OARS were examined in Iranian elderly. The aim of the present study was fulfilled by recruiting 482 individuals $>60$ years of age. Face, content, concurrent validities, and inter-rater and testretest reliabilities were examined. The Persian version of MFAQ/OARS exhibited an adequate face, content, and concurrent validity and reliability for functional assessment among Iranian elderly. 
Table 1. Demographic characteristics of the study sample $(n=482)$

\begin{tabular}{|c|c|c|c|}
\hline & Variable & Frequency & Percentage \\
\hline \multirow{3}{*}{ Gender } & Male & 269 & 55.8 \\
\hline & & & \\
\hline & Female & 213 & 44.2 \\
\hline \multirow{4}{*}{ Ethnicity } & Persian & 420 & 87.2 \\
\hline & Turk & 31 & 6.4 \\
\hline & Kurd & 20 & 4.1 \\
\hline & Lur & 11 & 2.3 \\
\hline \multirow{6}{*}{ Education } & $0-4$ years & 235 & 48.8 \\
\hline & $5-8$ years & 216 & 44.7 \\
\hline & Unfinished high school & 10 & 2.1 \\
\hline & High school diploma & 9 & 1.9 \\
\hline & Bachelor's degree & 7 & 1.5 \\
\hline & Master's degree & 5 & 1 \\
\hline \multirow{4}{*}{ Marital status } & Single & 25 & 5.2 \\
\hline & Married & 315 & 65.4 \\
\hline & Widowed & 110 & 22.8 \\
\hline & Divorced & 32 & 6.6 \\
\hline \multirow{5}{*}{ Living arrangement } & Only with partner & 195 & 40.5 \\
\hline & Alone & 164 & 34 \\
\hline & With partner and children & 117 & 24.3 \\
\hline & With a friend & 4 & 0.8 \\
\hline & Only with children & 2 & 0.4 \\
\hline
\end{tabular}

Iranian Rehabilitation Journal

Table 2. Correlations between MFAQ/OARS scores and those administered concurrently

\begin{tabular}{ccc}
\hline Concurrent Validity & Correlation & P \\
\hline Social resources & 0.45 & $\mathrm{P}<0.0001$ \\
Economic resources & 0.81 & $\mathrm{P}<0.0001$ \\
Mental health & 0.86 & $\mathrm{P}<0.0001$ \\
Physical health & 0.43 & $\mathrm{P}<0.0001$ \\
\hline activities of daily living & 0.86 & $\mathrm{P}<0.0001$ \\
\hline
\end{tabular}

Ilranian Rehabilitation Journa 
Table 3. Correlation between ratings

\begin{tabular}{cl}
\hline Domains & ICC \\
\hline Social resources & 0.96 \\
Economic resources & 0.97 \\
Mental health & 0.95 \\
Physical health & 0.89 \\
Activities of daily living & 0.78 \\
\hline
\end{tabular}

Iranian Rehabilitation Journal

The face validity of the MFAQ/OARS indicated the simplicity and clarity of the statements from the Iranian population. In the examination of content validly, all the questionnaire items were maintained, thereby confirming the content validity of the MFAQ/OARS.

The concurrent validity of the MFAQ/OARS in the elderly was examined using Spearman's rank correlation coefficient in each of the five domains. It was found to be moderate for social resources and physical health and high for economic resources, mental health, and activities of daily living $(>0.80)$.

\section{Discussion}

ICC was used to examine the inter-rater reliability for each of the five domains (0.78-0.97); the lowest and highest correlation coefficients indicated the activities of daily living and economic resources, respectively. However, regarding the inter-rater reliability of the original version of the questionnaire, the lowest ICC was for physical health (0.66), and the highest for everyday activities (0.86). Therefore, in both studies, the inter-rater reliability for all the five domains was reported as satisfactory [12]. The test-retest reliability of the questionnaire was also adequate for all the domains and subdomains. The test-retest reliability of the original questionnaire was reported as 90.7 [4]. According to the results obtained in this study, the validity and reliability were confirmed for Iranian elderly.

\section{Conclusion}

The MFAQ/OARS tool exhibits a satisfactory validity and reliability in assessing the functioning ability of Iranian elderly. It is a comprehensive instrument assessing different domains of functioning. On the other hand, it also compares between the overall score and different domain scores; therefore, researchers using this questionnaire can compare the respondent's scores on dif-

Table 4. Intraclass Correlation Coefficient (ICC)

\begin{tabular}{lcc}
\hline & Domains and Subdomains & ICC \\
\hline Social resources & Relationships & 0.98 \\
& Family & 0.77 \\
& Self-evaluation & 0.99 \\
& Social resources & 0.97 \\
Mental health & Mental capacity & 0.72 \\
& Psychological symptoms & 1 \\
& Mental health self-evaluation & 0.99 \\
\hline
\end{tabular}


ferent domains and take appropriate decisions about the care of the elderly based on the MFAQ/OARS scores.

The MFAQ/OARS tool can be used for hospitalized elderly, as well as, those residing in retirement homes. However, the present study was only focused on the elderly living in the community. Therefore, future studies are essential to assess the psychometric properties of the questionnaire in the other populations of older individuals. Nevertheless, the present study had some limitations considering the economic differences between Iran and the USA. Thus, future studies should encompass the opinions of economic experts. The validity in the economic domain could be improved by considering the level of inflation and poverty line and accurate categorization of income levels. Finally, we suggest that the MFAQ/OARS should be used in functional assessments of the elderly and determining the level of optimal care.

\section{Ethical Considerations}

\section{Compliance with ethical guidelines}

This article was approved by the Ethics Committee at the University of Social Welfare and Rehabilitation Sciences (Number: IR.USWR.REC.1395.235). The informed consent was obtained for participation in the study. The study objectives were explained to the participants by the researchers.

\section{Funding}

This study was not supported by a specific grant from any funding agency, and hence, was funded by the first author. It was extracted from a Master's thesis (Number: 941193014) by the first author in Department of Aging, University of Social Welfare and Rehabilitation Sciences, Tehran, Iran.

\section{Conflict of interest}

The authors declare no conflict of interest.

\section{Acknowledgments}

The authors sincerely thank Dr. Hosseinzadeh, the translators, and all those who helped in this project.

\section{References}

[1] Shirazi Khah M, Mousavi M, Sahaf R. [Study of health and social indicators of elderly women in Iran (Persian)]. Iranian Journal of Ageing. 2012; 6(S1):66-78

[2] Amini R ISR, Sahaf R. Aging in Iran: past, present and future. Journal of Aging in Emerging Economies. 2013; 4(1):1734.

[3] Arking R. Biology of aging: Observations and principles Oxford: Oxford University Press; 2006.

[4] World Health Organization. International classification of functioning, disability and health (ICF). Geneva: World Health Organization; 2001.

[5] Halter J, Ouslander J, Tinetti M, Studenski S, High K, Asthana S. Hazzard's geriatric medicine and gerontology. New York: McGraw-Hill; 2009.

[6] Üstün TB, Chatterji S, Kostansjek N, Bickenbach J. WHO's ICF and functional status information in health records. Health Care Financing Review. 2003; 24(3):77. [PMID] [PMM CID]

[7] Katz S, Ford AB, Moskowitz RW, Jackson BA, Jaffe MW. Studies of illness in the aged: the index of ADL: a standardized measure of biological and psychosocial function. JAMA. 1963; 185(12):914-9. [DOI:10.1001/jama.1963.03060120024016] [PMID]

[8] Oveisgharan S, Shirani S, Ghorbani A, Soltanzade A, Baghaei A, Hosseini S, et al. Barthel index in a Middle-East country: Tanslation, validity and reliability. Cerebrovascular Diseases. 2006; 22(5-6):350-4. [DOI:10.1159/000094850] [PMID]

[9] Graf C. The Lawton instrumental activities of daily living (IADL) scale. Medsurg Nursing. 2009; 18(5):315-6. [PMID]

[10] Üstün TB, Chatterji S, Kostanjsek N, Rehm J, Kennedy C, Epping-Jordan J, et al. Developing the World Health Organization disability assessment schedule 2.0. Bulletin of the World Health Organization. 2010; 88(11):815-23. [DOI:10.2471/BLT.09.067231] [PMID] [PMCID]

[11] Rajabi G, Namadmalan M, Ghasemzadeh R, Foroughan M, Zahednejad S, Dijkstra A. [Psychometric properties of the Persian version of care dependency scale in older people nursing home residents (Persian)]. Payesh. 2015; 14(2):193-204.

[12] Fillenbaum GG. Multidimensional functional assessment of older adults: The Duke Older Americans Resources and Services procedures. London: Psychology Press; 2013.

[13] Burholt V, Windle G, Ferring D, Balducci C, Fagerström C, Thissen F, et al. Reliability and validity of the Older Americans Resources and Services (OARS) social resources scale in six European countries. The Journals of Gerontology Series B: Psychological Sciences and Social Sciences. 2007; 62(6):S371S9. [DOI:10.1093/geronb/62.6.S371] [PMID]

[14] Blay SL, Ramos LR, Mari JJ. Validity of a Brazilian version of the Older Americans Resources and Services (OARS) mental health screening questionnaire. Journal of the American Geriatrics Society. 1988; 36(8):687-92. [DOI:10.1111/j.1532-5415.1988.tb07169.x]

[15] Grau FG, Eiroa PP, Cayuela DA. Spanish version of the OARS Multidimensional Functional Assessment Question- 
naire: Cross-cultural adaptation and validity measurement. Atención Primaria. 1996; 17(8):486-95.

[16] Rodrigues RMC. [Validation of the European Portuguese version of the Older Americans Resources and Services instrument (Spanish)]. Pan American Journal of Public Health. 2008; 23(2):109-15.

[17] Bullinger M, Alonso J, Apolone G, Leplège A, Sullivan M, Wood-Dauphinee $S$, et al. Translating health status questionnaires and evaluating their quality: the IQOLA project approach. Journal of Clinical Epidemiology. 1998; 51(11):913-23. [DOI:10.1016/S0895-4356(98)00082-1]

[18] Salavati M, Mazaheri M, Khosrozadeh F, Mousavi SME, Negahban H, Shojaei H. The Persian version of locomotor capabilities index: translation, reliability and validity in individuals with lower limb amputation. Quality of Life Research. 2011; 20(1):1-7. [DOI:10.1007/s11136-010-9716-3] [PMID]

[19] Yaghmaei F. [Critical review of psychometric properties in research questionnaires (Persian)]. Faculty of Nursing of Midwifery Quarterly. 2006; 16(52): 66-75.

[20] Abedzadeh-kalahroudi M, Razi E, Sehat M, Asadi-Lari M. Psychometric properties of the world health organization disability assessment schedule II-12 Item (WHODAS II) in trauma patients. Injury. 2016; 47(5):1104-8. [DOI:10.1016/j. injury.2015.11.046] [PMID]

[21] Malakouti K FP, Mirabzadeh A, Salavati M, Kahani S. [Validation of Geriatric Depression Scale (GDS-15) in Iran (Persian)]. Research in Medicine. 2006; 30(4):361-9.

[22] Yesavage JA, Sheikh JI. 9/Geriatric Depression Scale (GDS) recent evidence and development of a shorter version. Clinical Gerontologist. 1986; 5(1-2):165-73. [DOI:10.1300/ J018v05n01_09] 
